\section{A novel classification of hematologic conditions in patients with Fanconi anemia}

Patients with the inherited bone marrow failure (BMF) syndrome Fanconi anemia (FA) have an increased risk of myelodysplastic syndrome (MDS) and acute myeloid leukemia (AML). ${ }^{1,2}$ The bone marrow (BM) in FA patients is typically hypocellular and it can mimic that of other conditions such as acquired refractory cytopenia of childhood. ${ }^{3}$ In patients with FA, the diagnosis of MDS cannot be made based on the presence of cytopenia and myelodysplastic features alone (with the exception of distinct subtypes such as cases with a rapid rise in cellularity in which MDS needs to be considered), but requires an elevated blast count and/or other definite signs of transformation, such as duplication of chromosome $3 q$ $(3 q+)$, deletion $7 q(7 q-)$, or monosomy $7(-7),{ }^{4-6}$ aberrations known to be associated with a poor prognosis in FA patients. ${ }^{5,7}$ In contrast, in the absence of an elevated blast percentage, not every clonal aberration warrants the diagnosis of MDS because not all aberrations, such as duplication of chromosome 1q $(1 \mathrm{q}+)$, deletion $20 \mathrm{q}$ $(20 q-)$, or deletion $6 p(6 p-)$, are associated with transformation. ${ }^{5,6,9-11}$ It is increasingly recognized that FA patients display clonal mosaicism generally correlated with a more common occurrence of hematologic neoplasia and poorer prognosis. ${ }^{11}$

An internationally agreed classification for the diagnosis of hematologic conditions in patients with FA is lacking, but a classification system is important for the clinical care and treatment of patients with this rare disease. Here, we propose an algorithm for the diagnosis of benign and malignant hematologic conditions in FA that is based on blast percentage and cytogenetics (Figure 1). Within this classification, we propose the term "aberration of indeterminate potential in patients with FA" (FAAIP), to classify patients with clonal aberrations, such as duplication of $1 \mathrm{q}$, which remain stable over a prolonged period without transformation. The classification includes: (i) FA-BMF, defined by normal blast percentage and normal cytogenetics in a patient with cytopenia; (ii) FA-AIP, defined by normal blast percentage and AIP; (iii) FA-MDS-without excess blasts or FA-MDS-non-EB, defined by normal blast percentage and clonal aberrations unambiguously associated with transformation; (iv) FA-MDS with excess blasts or FA-MDS-EB, defined by increased blast percentage in $\mathrm{BM} \geq 5 \%$ to $<20 \%$ or in peripheral blood (PB) $\geq 2 \%$ to $<20 \%$, (but $\mathrm{BM}$ and $\mathrm{PB}$ $<20 \%$ blasts); and (v) FA-AML, defined by increased blast percentage in $\mathrm{BM}$ or $\mathrm{PB} \geq 20 \%$. One main intention of $\mathrm{BM}$ surveillance is to identify patients with FA-MDS-non EB prior to transformation with elevated blast count (FAMDS-EB or FA-AML) in order to increase overall survival following early hematopoietic stem cell transplantation.

We used this diagnostic algorithm to analyze data from 86 patients enrolled in the German FA registry (FAR01)/ Cancer Predisposition Registry (DRKS00017382). FAMDS-non EB and FA-MDS-EB patients were also enrolled in the registry of the European Working Group of Myelodysplastic Syndrome in Childhood (EWOGMDS). The study was approved by the ethical review boards of Hannover Medical School and Freiburg University. Patients were analyzed cytogenetically as part of their clinically indicated cancer and BMF surveillance program. Cytogenetic analyses were performed in our reference laboratory on $\mathrm{BM}$ aspirates by karyotyping and fluorescence in situ hybridization. In 62 of the 86 patients, serial cytogenetic analyses were performed (mean, 4.9 analyses per patient). The fluorescence in situ hybridization analyses were employed to identify $3 q+, 7 q-,-7$, or (cryptic) RUNX1 (AML1) aberrations. BM cytology and pathology were reviewed centrally.

Clinical characteristics as well as all cytogenetic followup examinations of the 86 FA patients are shown in Table 1 and Online Supplementary Tables S1-S3. Out of the 86 FA patients, $60(70 \%)$ had a normal blast percentage and a normal karyotype at every time point. All of these patients were classified as having FA-BMF. There were no patients with an elevated blast percentage in $\mathrm{PB}$ and/or BM who had a normal karyotype. Twenty-six patients showed acquired clonal chromosomal aberrations (30\%) (Table 1). The most frequent chromosomal aberration

Table 1. Clinical characteristics of patients with Fanconi anemia $(n=86)$.

\begin{tabular}{|c|c|}
\hline Characteristics & n (\%) \\
\hline Age at FA diagnosis ${ }^{\mathrm{a}}$ & 8 years \\
\hline median (range) & (1 month - 25 years) \\
\hline Age at last follow up & 13 years \\
\hline median (range) & (2 - 43 years) \\
\hline Death $^{b}$ & $7(8 \%)$ \\
\hline \multirow[t]{2}{*}{ Age at death } & median (range) \\
\hline & 4 years ( 3 - 43 years) \\
\hline $\mathrm{HSCT}^{\mathrm{c}}$ & $39(45 \%)$ \\
\hline Age at HSCT & 9 years \\
\hline median (range) & (3- 42 years) \\
\hline \multicolumn{2}{|l|}{ Sex } \\
\hline Female & $38(44 \%)$ \\
\hline Male & $48(56 \%)$ \\
\hline \multicolumn{2}{|l|}{ Complementation group } \\
\hline FANCA & $42(49 \%)$ \\
\hline FANCC & $8(9 \%)$ \\
\hline FANCD2 & $4(5 \%)$ \\
\hline FANCP & $3(3 \%)$ \\
\hline FANCB & $2(2 \%)$ \\
\hline FANCJ & $2(2 \%)$ \\
\hline FANCL & $2(2 \%)$ \\
\hline FANC:D1, G, I, T (each) & $1(1 \%)$ \\
\hline Unknown & $20(23 \%)$ \\
\hline Tranformation to AML/MDS & $15(17 \%)$ \\
\hline Age at tranformation to FA-MDS & 11 years \\
\hline median (range) & (3- 42 years) \\
\hline FA-MDS-non EB & $8(9 \%)$ \\
\hline FA-MDS-EB & $2(2 \%)$ \\
\hline FA-AML & $5(6 \%)$ \\
\hline FA-AIP & $10(12 \%)$ \\
\hline \multicolumn{2}{|l|}{ Karyoytype } \\
\hline Abnormal & $26(30 \%)$ \\
\hline Patients with transformation to AML/MDS & $15(17 \%)$ \\
\hline Normal & $60(70 \%)$ \\
\hline Patients with transformation to AML/MDS & 0 \\
\hline
\end{tabular}

${ }^{\mathrm{a}}$ Date of diagnosis of Fanconi anemia not known for five patients. ${ }^{\mathrm{b}} \mathrm{Date}$ of death not known for four patients. ${ }^{c}$ Date of transplantation not known for five patients ${ }^{\mathrm{d}}$ Date of transformation not known for two patients. FA: Fanconi anemia; HSCT hematopoietic stem cell transplantation; AML: acute myeloid leukemia; MDS myelodysplastic syndrome; EB: excess blasts; AIP: aberration of indeterminate potential. 
was a $1 q+(14 \%)$ followed by $3 q+(9 \%), 7 q-(9 \%),-7$ $(3 \%), 7 \mathrm{p}-(3 \%)$, and $6 \mathrm{p}-(3 \%)$ (Online Supplementary Table S2). Fifteen of these 26 patients $(58 \%)$ were diagnosed with a myeloid neoplasia: Eight patients were categorized as having FA-MDS-non EB (FA2, 6, 13, 54, 59, 70, 79 , and 89), two patients as having FA-MDS-EB (FA3 and 12) and five patients met the criteria for FA-AML (FA27 $39,43,61$, and 76). Another ten patients were diagnosed with FA-AIP (FA22, 25, 26, 35, 45, 47, 77, 84, 86, and 87). In patients with FA-related myeloid neoplasia, we observed the expected recurrent pattern of chromosomal aberrations (e.g., 3q+, 7q-, -7). Patients with FA-AIP had a different pattern of chromosomal aberrations, such as $1 \mathrm{q}+, 6 \mathrm{p}-$, and $7 \mathrm{p}-$ (Online Supplementary Tables S1 and S3). Furthermore, patients with FA-AML had different cytogenetic aberrations than patients with de novo AML, which is consistent with previous findings. ${ }^{12}$ Our dataset was too small to correlate FA-complementation subgroups with hematologic findings.
To further investigate the chronological sequence of aberrations in FA-AIP, FA-MDS-non EB, FA-MDS-EB, and FA-AML over a prolonged period, we analyzed the follow-up examinations of the 26 patients with chromosomal aberrations over the course of several years (Figure 2, Online Supplementary Table S1). Twelve of 26 patients showed $1 \mathrm{q}+$, isolated or in combination with additional aberrations (FA2, 6, 12, 22, 26, 27, 47, 70, 79, 84, 86, and 87). Only patients with $1 \mathrm{q}+$ in combination with $3 \mathrm{q}+$ or 7q- developed myeloid neoplasia (FA2, 6, 12, 27, 70, and 79 ), while patients with $1 \mathrm{q}+$ isolated or in combination with other aberrations (e.g., 6p- or 7p-) did not progress to develop a myeloid neoplasia within the observation period (FA22, 26, 47, 84, 86, and 87) (Figure 2). Several patients displayed a stable clone without transformation for several years. The presence of the recurrent aberrations $3 \mathrm{q}+, 7 \mathrm{q}-/-7$ or a complex karyotype was always indicative of myeloid neoplasm (FA2, 3, 6, 12, 13, 27, 39, $43,54,59,61,70,79$, and 89) with the exception of one

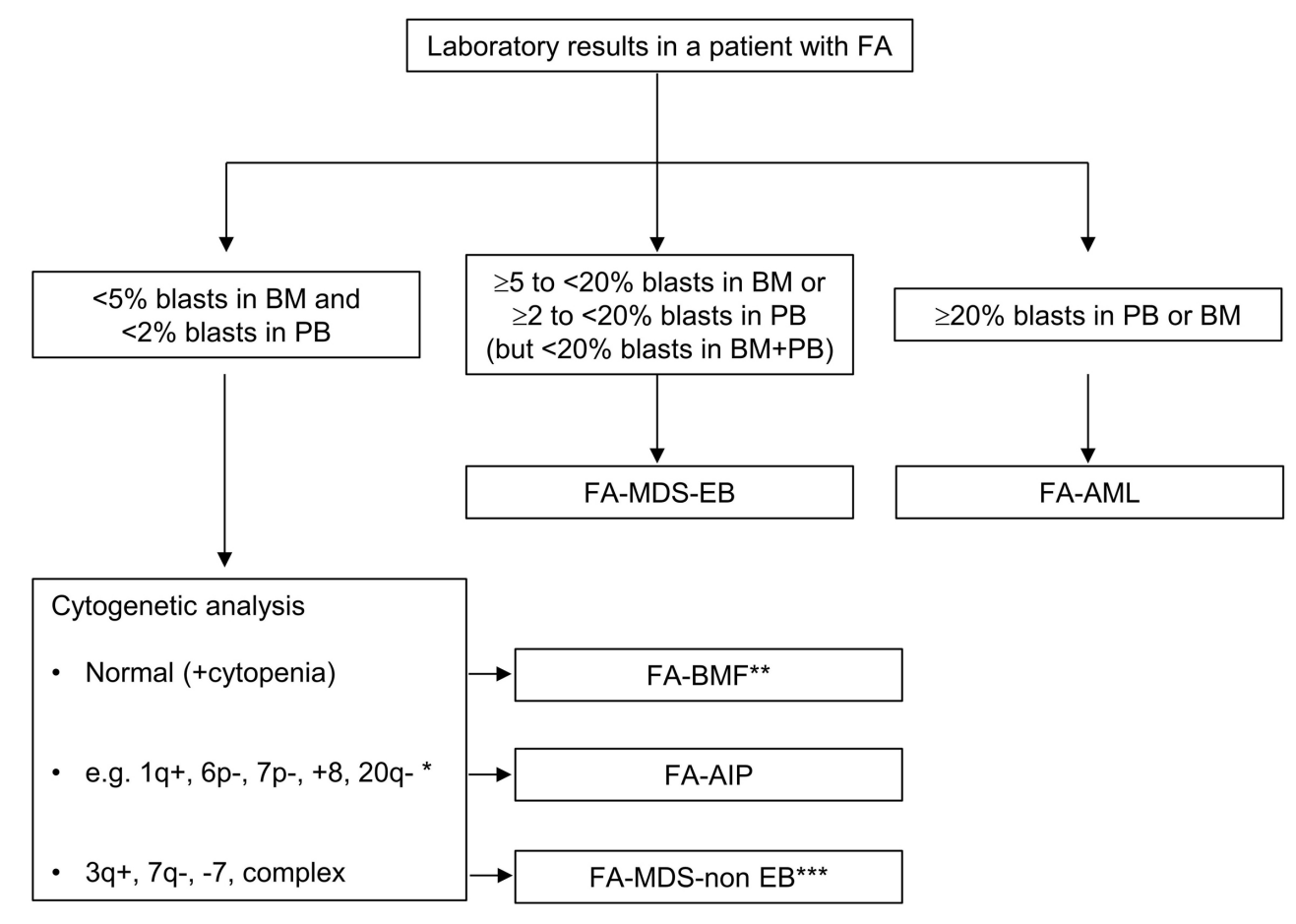

\begin{tabular}{|c|c|c|c|c|}
\hline \multicolumn{5}{|c|}{ Spectrum of hematologic conditions in patients with FA } \\
\hline FA-BMF & FA-AIP & FA-MDS-non EB & FA-MDS-EB & FA-AML \\
\hline
\end{tabular}

* all cytogenetic aberrations (exclusion: $3 q+, 7 q-,-7$, complex)

${ }^{* *}$ in cases with rapid rise in BM cellularity FA-MDS needs to be considered

*** in pediatric patients generally Refractory Cytopenia of Childhood (RCC)

Figure 1. Proposed diagnostic algorithm for hematologic conditions in patients with Fanconi anemia. FA: Fanconi anemia; BM: bone marrow; PB; peripheral blood; FA-MDS-EB: myelodysplastic syndrome with excess blasts in a patient with FA; FA-AML: acute myeloid leukemia in a patient with FA; FA-BMF: BM failure in a patient with FA, a normal blast percentage and normal cytogenetics; FA-AIP: aberration of indeterminate potential in a patient with FA; FA-MDS-non-EB: myelodysplastic syndrome without excess blasts in a patient with FA. 


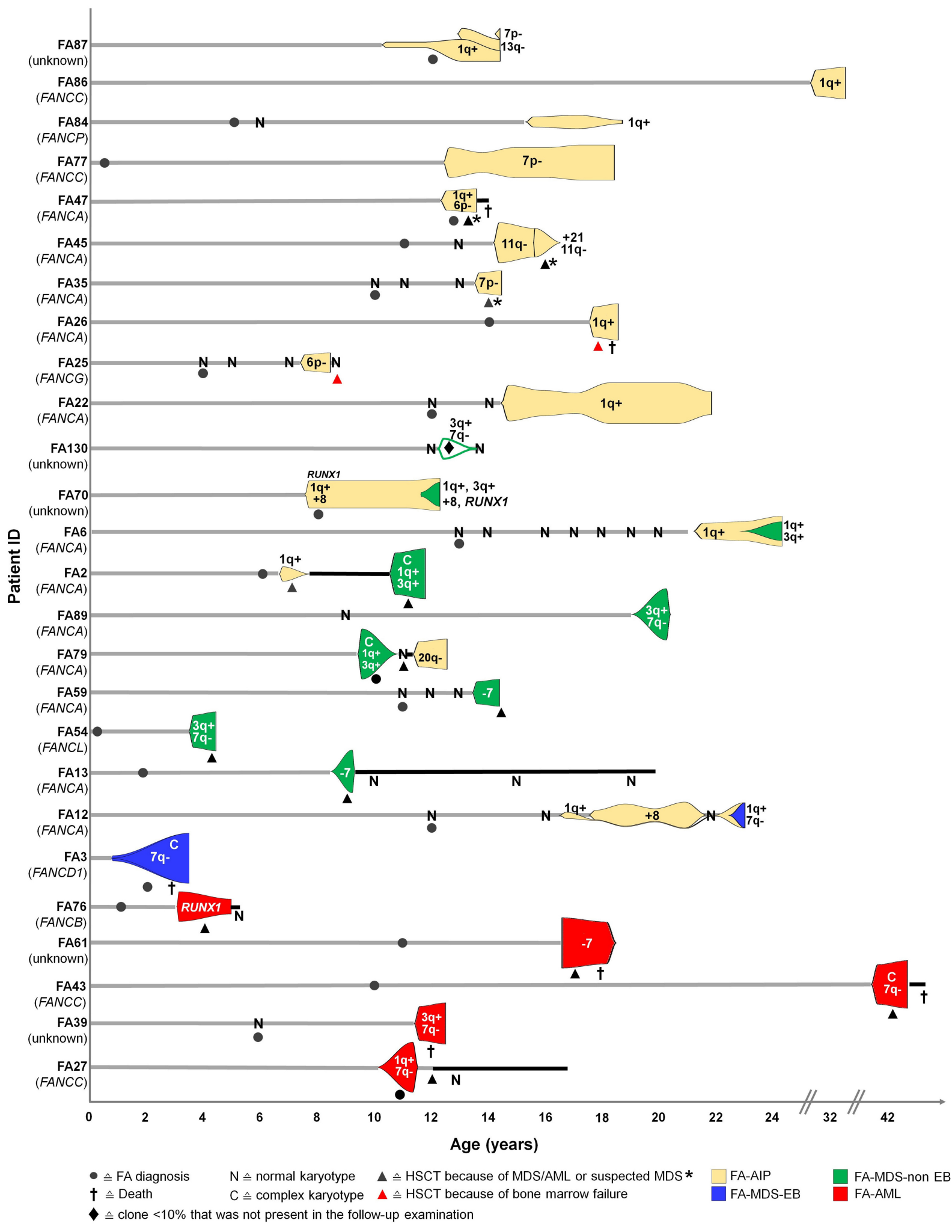

Figure 2. Chronology of recurrent cytogenetic events in Fanconi anemia with myelodysplastic syndrome or aberrations of indeterminate potential ( $\mathrm{n}=26)$. Each bar represents cytogenetic follow-up over the course of several years (recurrent aberration, clone size) as well as additional clinical information. Additional nonrecurrent clonal aberrations of these patients are shown in Online Supplementary Table S2. Patient FA130 showed cytogenetic aberrations associated with myeloid neoplasia in $<10 \%$ of cells, and no blasts in peripheral blood or bone marrow, which were no longer detectable in the following examination that revealed a normal karyotype. To construct the clone size over time we used the "clonal evolution plot R". ${ }^{14}$ FA: Fanconi anemia; HSCT: hematopoietic stem cell transplantation; MDS: myelodysplastic syndrome; AML: acute myeloid leukemia; AIP: aberration of indeterminate potential; EB: excess blasts. 
case (FA130): This patient had a small clone ( $9 \%$ of cells) with $3 q+$ and $7 q$ - (Figure 2, Online Supplementary Table S1) which was no longer detectable in the following examination that revealed a normal karyotype. This observation may indicate that the presence of a small clone with aberrations affecting $<10 \%$ of analyzed cells may not be sufficient to establish the diagnosis of FAMDS in all cases. Thus, short-term repeat analysis is recommended to avert unnecessary hematopoietic stem cell transplantation. Clonal evolution (8/15 vs. $1 / 10)$ as well as a complex karyotype (5/15 vs. $0 / 10)$ occurred more frequently in patients with myeloid neoplasia $(n=15)$ than in patients with FA-AIP $(\mathrm{n}=10)(P<0.05$, Fisher exact test) (Online Supplementary Table S2). In two patients with FAAIP (FA35 and 87) and in three patients with myeloid neoplasms (FA2, 12, and 79), the original clone was either replaced by a new clone or at least one new clone appeared in addition to the original clone. Apart from patient FA130, in one patient with FA-AIP (FA25) and in one FA patient with myeloid neoplasia (FA12) the aberrant clone disappeared and the patients showed a normal karyotype during follow-up examinations. One patient (FA79) developed a 20q- aberration after hematopoietic stem cell transplantation. Notably, this patient had incomplete donor chimerism. Four out of ten patients with FA-AIP (FA2, 6, 12, and 70) developed a myeloid neoplasm over time within the observation period. This finding indicates that the presence of FA-AIP is not a predictor for rapid transformation. It is conceivable that additional remaining patients may transform after a longer time period. Cryptic RUNX1 aberrations were not unambiguously associated with immediate transformation, as noted in patient FA70 who developed FA-MDSnon-EB approximately 2 years after this lesion had been detected. Another patient with a RUNX1 aberration developed FA-AML (FA76). Larger cohorts are required to determine the prognostic value of this aberration and whether immediate hematopoietic stem cell transplantation is indicated because of an isolated $R U N X 1$ aberration in a FA patient.

In conclusion, we propose an algorithm for the diagnosis of benign and malignant hematologic conditions in patients with FA, which may serve as a model for other syndromes predisposing to MDS and AML. The concept of AIP may also apply to other inherited BMF syndromes such as Shwachman-Diamond syndrome, since patients with this syndrome commonly develop cytogenetic abnormalities including deletion $20 \mathrm{q}$ and isochromosome $7 \mathrm{q}$ that are not associated with a high risk of progression to MDS. ${ }^{13}$

\footnotetext{
Yvonne Lisa Behrens, ${ }^{1 *}$ Gudrun Göhring, ${ }^{1^{*}}$ Randa Bawadi, ${ }^{1}$ Sümeyye Cöktü, ${ }^{2}$ Christina Reimer, ${ }^{2}$ Beatrice Hoffmann, ${ }^{2}$ Birte Sänger, ${ }^{2}$ Simon Käfer, ${ }_{1}^{1}$ Felicitas Thol, ${ }^{3}$ Miriam Erlacher, ${ }^{4,5,6}$ Charlotte M. Niemeyer, ${ }^{4,5,6}$ Irith Baumann, ${ }^{7}$ Reinhard Kalb, ${ }^{8}$ Detlev Schindler ${ }^{8}$ and Christian Peter Kratz ${ }^{2}$

${ }^{*} Y L B$ and GG contributed equally as co-first authors.

${ }^{1}$ Department of Human Genetics, Hannover Medical School, Hannover; ${ }^{2}$ Pediatric Hematology and Oncology, Hannover Medical School, Hannover; ${ }^{3}$ Department of Hematology, Hemostasis, Oncology and Stem Cell Transplantation, Hannover Medical School, Hannover; ${ }^{4}$ Division of Pediatric Hematology and Oncology, Department of Pediatrics and Adolescent Medicine, University Medical Center Freiburg, Faculty of Medicine, University of Freiburg, Freiburg; ${ }^{5}$ German Cancer Consortium (DKTK), Freiburg; 6 German Cancer Research Center (DKFZ), Heidelberg; Institute of Pathology, Kaufbeuren and ${ }^{8}$ Department of Human Genetics, University of Würzburg, Biocenter, Würzburg, Germany
}

Correspondence:

CHRISTIAN P.KRATZ - kratz.christian@mh-hannover.de

doi:10.3324/haematol.2021.279332

Received: May 28, 2021.

Accepted: June 22, 2021.

Pre-published: July 1, 2021.

Disclosures: no conflicts of interest to disclose.

Contributions: the study was conceived by $C P K, Y L B$, and GG; data and material of the study was generated by $Y L B, G G, R K$, $D S, I B, M E, C N, S C, B S, C R, B H, S K, C P K$ and $R B$; data collection was performed by $Y L B, G G, C P K$; interpretation and analysis were conducted by $Y L B, G G, C P K, M E, C N, I B$ and FT; YLB, GG, CN, and CPK wrote the manuscript; $F A$ diagnostics and complementation analysis was done by $D S$ and $R K$; the paper was edited by all authors.

All authors have read and approved the final manuscript.

Acknowledgments: the authors thank the members of the Deutsche Fanconi Hilfe e.V. for their support.

Funding: CPK, GG and FT have been supported by the BMBF ADDRess (01GM1909A), DS und RK by BMBF ADDRess (01GM1909B), GG, CN and ME by the BMBF MyPred (01GM1911A). CPK has been supported by the Deutsche Kinderkrebsstiftung (DKS2019.13).

\section{References}

1. Alter BP, Giri N, Savage SA, Rosenberg PS. Cancer in the National Cancer Institute inherited bone marrow failure syndrome cohort after fifteen years of follow-up. Haematologica. 2018;103(1):30-39.

2. Tsai FD, Lindsley RC. Clonal hematopoiesis in the inherited bone marrow failure syndromes. Blood. 2020;136(14):1615-1622.

3. Yoshimi A, Niemeyer C, Baumann I, et al. High incidence of Fanconi anaemia in patients with a morphological picture consistent with refractory cytopenia of childhood. Br J Haematol. 2013;160(1):109111.

4. Tonnies H, Huber S, Kuhl JS, Gerlach A, Ebell W, Neitzel H. Clonal chromosomal aberrations in bone marrow cells of Fanconi anemia patients: gains of the chromosomal segment 3q26q29 as an adverse risk factor. Blood. 2003;101(10):3872-3874.

5. Peffault de Latour R, Soulier J. How I treat MDS and AML in Fanconi anemia. Blood. 2016;127(24):2971-2979.

6. Quentin S, Cuccuini W, Ceccaldi R, et al. Myelodysplasia and leukemia of Fanconi anemia are associated with a specific pattern of genomic abnormalities that includes cryptic RUNX1/AML1 lesions. Blood. 2011;117(15):e161-170.

7. Meyer S, Neitzel H, Tonnies H. Chromosomal aberrations associated with clonal evolution and leukemic transformation in fanconi anemia: clinical and biological implications. Anemia. 2012; 2012:349837.

8. Wang Y, Zhou W, Alter BP, et al. Chromosomal aberrations and survival after unrelated donor hematopoietic stem cell transplant in patients with Fanconi anemia. Biol Blood Marrow Transplant. 2018; 24(10):2003-2008.

9. Soulier J. Fanconi anemia. Hematology Am Soc Hematol Educ Program. 2011;2011:492-497.

10. Mehta PA, Harris RE, Davies SM, et al. Numerical chromosomal changes and risk of development of myelodysplastic syndrome-acute myeloid leukemia in patients with Fanconi anemia. Cancer Genet Cytogenet. 2010;203(2):180-186.

11. Reina-Castillon J, Pujol R, Lopez-Sanchez M, et al. Detectable clonal mosaicism in blood as a biomarker of cancer risk in Fanconi anemia. Blood Adv. 2017;1(5):319-329.

12. Rochowski A, Olson SB, Alonzo TA, Gerbing RB, Lange BJ, Alter BP. Patients with Fanconi anemia and AML have different cytogenetic clones than de novo cases of AML. Pediatr Blood Cancer. 2012; 59(5):922-924.

13. Myers KC, Furutani E, Weller E, et al. Clinical features and outcomes of patients with Shwachman-Diamond syndrome and myelodysplastic syndrome or acute myeloid leukaemia: a multicentre, retrospective, cohort study. Lancet Haematol. 2020;7(3):e238-e246.

14. Miller CA, McMichael J, Dang HX, et al. Visualizing tumor evolution with the fishplot package for R. BMC Genomics. 2016; 17(1):880. 